\title{
Targeted Metabolite Profiling: Sample Preparation Techniques for GC-MS- Based Steroid Analysis
}

\author{
Krishna Chaitanya Sadanala ${ }^{\mathrm{a}, \mathrm{b}}$, Jeongae Lee ${ }^{\mathrm{a}}$, Bong Chul Chung ${ }^{\mathrm{a}, \mathrm{b}}$, and Man Ho Choi ${ }^{\mathrm{a}, \mathrm{b} *}$ \\ ${ }^{a}$ Future Convergence Research Division, Korea Institute of Science and Technology, Seoul 136-791, Korea \\ ${ }^{b}$ Department of Biomolecular Science, University of Science and Technology, Daejeon 305-333, Korea
}

Received March 3, 2012; Revised March 7, 2012; Accepted March 7, 2012

First published on the web March 15, 2012; DOI: 10.5478/MSL.2012.3.1.004

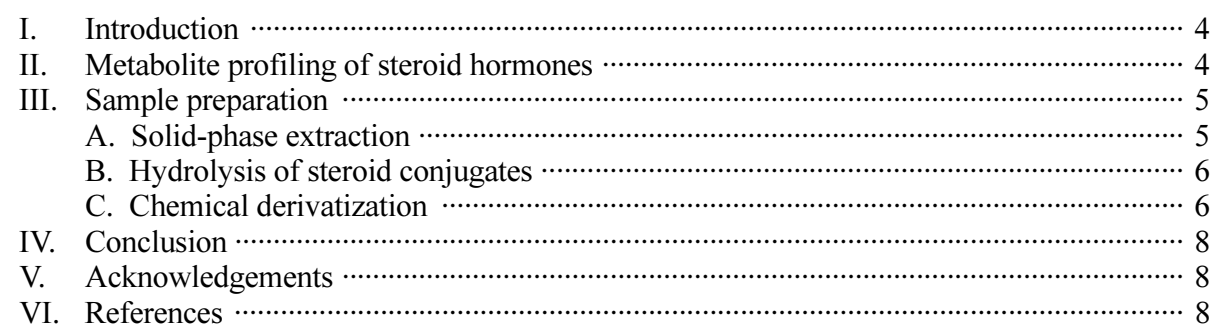

Key words: Steroids, GC-MS, Solid-phase extraction, Enzymatic hydrolysis, Silylation, Acylation

\section{Introduction}

Many clinical events result in specific and characteristic alterations that originate in biochemical imbalances and are measurable in biological fluids and tissues. Metabolomics consists of the qualitative and quantitative analysis of intraand intercellular metabolites using 2 different analytical methods: (1) non-targeted metabolite profiling and (2) targeted metabolite profiling. Non-targeted metabolite profiling involves the identification and characterization of a large number of metabolites and their precursors. In contrast, targeted metabolite profiling focuses on quantitative changes in metabolites of interest (e.g., amino acids, carbohydrates, steroids, and fatty acids) based on a priori knowledge of the biological function or metabolic pathway.

Steroid hormones regulate human body functions and are components of the endocrine system, which coordinates physiological and behavioral responses for specific biological purposes, such as reproduction. Steroids are active at very low concentrations and have a range of biological effects (e.g., homeostasis). Steroid analysis has gained importance in biomedical, pharmaceutical, engineering, and agricultural sciences. Clinical assays for steroid analysis must be robust, highly sensitive, automated, and simple.

Steroids analysis is used in the clinical events for the diagnosis of stress, ${ }^{1}$ Cushing's syndrome, ${ }^{2}$ hirsutism, ${ }^{3}$

*Reprint requests to Dr. Man Ho Choi

E-mail:mh_choi@kist.re.kr congenital steroid enzyme deficiency, ${ }^{4}$ hypertension, ${ }^{5}$ and as a doping test. ${ }^{6}$ Biological specimens used in clinical applications include urine, serum, and saliva, and they exhibit some behaviors of steroids (e.g., free forms, conjugated forms, protein-binding forms). Steroids in biological specimens behave differently and different considerations are required when developing methods for their analysis. ${ }^{7}$ Due to its high sensitivity and selectivity, gas chromatographymass spectrometry (GC-MS) is used to analyze metabolites and precursors of steroid hormones. In recent, GC-MSbased steroid phenol-typing has demonstrated diagnostic power and the potential to discover metabolic diseases. ${ }^{8}$

This review focuses on the methods involved in a metabolomic study based on GC-MS analysis of steroid hormones in biological specimens. Sample preparation procedures and their major steps are discussed in detail. The various techniques and materials that have been used to achieve efficient analytical results during the past decade are described. Comparative studies of crucial aspects are included to facilitate the understanding of the developed methods.

\section{Metabolite Profiling of Steroid Hormones}

Metabolomics provides comprehensive information on the metabolic state of biological systems by detecting bioactive metabolites. It establishes the relationship between phenotype and metabolism, which is a key aspect in connecting the distribution of metabolites with their 
biological functions. ${ }^{9,10}$ Metabolite profiling can help to understand disease mechanisms, identify new diagnostic markers, and increase the ability to predict biological variations. ${ }^{11}$

Many naturally occurring steroids with similar chemical structures could produce biological changes. Endogenous steroids are divided into 5 groups: androgens, estrogens, corticoids, progestins, and sterols, which are generally synthesized from cholesterol in the adrenal cortex, ovaries, and testes. These steroids play a variety of important physiological roles in peripheral target tissues or the central nervous system. ${ }^{12}$ Steroid metabolism is important for the production of these hormones and the regulation of their cellular physiological actions. The steroid metabolic pathways involve 2 major types of enzymes: cytochrome P450 and other steroid oxidoreductases. Many endocrine disorders can be attributed to defects in these enzymes, which lead to hormonal imbalance and serious consequences. ${ }^{13-19}$

Mass spectrometry (MS) has been used to simultaneously characterize a large number of metabolites in complex living systems, ${ }^{20}$ and it focuses on local metabolisms to decipher functions and metabolic alterations. MS-based metabolomics have therefore become a promising technique in clinical diagnosis. ${ }^{21,22}$ The GC-MS methods offer high chromatographic resolution as well as the ability to identify unknown compounds from public libraries. ${ }^{23}$ Therefore, GC-MS profiling has been widely used for steroid analysis, ${ }^{24-27}$ and provides the basis for techniques that can be applied to large-scale clinical studies. ${ }^{28}$

\section{Sample Preparation}

The isolation of steroids from complex biological specimens is a prerequisite for GC-MS analysis. The solid-phase extraction (SPE) method is preferentially used when low sample complexity is required for the simultaneous detection of urinary steroids in both screening and confirmation analyses. These methods are also combined with enzymatic hydrolysis and additional liquid-liquid extraction (LLE) steps to extract unconjugated steroids. ${ }^{29}$ Many steroids are heat labile and must be derivatized before analysis to avoid compound decomposition and to improve their chromatographic performance. ${ }^{30}$

\section{Solid-phase extraction}

SPE has become the comprehensive technique of choice for simultaneous extraction and concentration of many compounds in aqueous samples. ${ }^{31,32}$ SPE methods can be limited by the difficulty in choosing from a large variety of SPE sorbents and by the different chemical properties in simultaneous analysis of steroids. In SPE experiments, 4 hydrophilic sorbents were prepared: Oasis $\operatorname{HLB}^{\mathrm{TM}}(3 \mathrm{~mL}$, $60 \mathrm{mg}$; Waters, Milford, MA, USA), Sep-pak ${ }^{\mathrm{TM}} \mathrm{C} 18$ (3 mL, $200 \mathrm{mg}$; Waters), Serdolit ${ }^{\mathrm{TM}}$ PAD-1 (0.1 0.2 mm analytical grade; Serva, Hiedelberg, Germany), and Sep-pak ${ }^{\mathrm{TM}}$ amino- propyl ( $\mathrm{NH}_{2}: 3 \mathrm{~mL}, 200 \mathrm{mg}$; Waters). Low recoveries were obtained with the Sep-pak C18, amino-propyl, and Serdolit PAD-1 columns, whereas the Oasis HLB cartridge increased the recoveries. This is consistent with the observation that Oasis HLB provides selective isolation and enrichment of polar steroids, such as corticoids and estrogens, from complex biological specimens. ${ }^{34,36}$ Furthermore, the Oasis HLB cartridge contains a unique copolymer sorbent with hydrophilic and lipophilic groups in proportions that allow high and reproducible recoveries of many compounds. ${ }^{33-39}$ In addition, the Oasis HLB can be used to extract steroids from various sample matrices, including urine, ${ }^{40-46}$ serum, ${ }^{47}$ plasma, ${ }^{48}$ cardiac tissue, ${ }^{49}$ and hair extract. ${ }^{50}$ Among the SPE cartridges tested, amino-propyl had lower sample recoveries than the other SPE cartridges. ${ }^{30}$ Serdolit PAD-1 slurry was used in the simultaneous detection of 9 androgen glucuronides in urine specimens. ${ }^{51}$

As an alternative SPE technique, a column with an inclusion complex-based $\beta$-cyclodextrin $(\beta-\mathrm{CD})$ sorbent has been used in GC-MS analysis of 45 endogenous steroids. ${ }^{52}$ The GC-MS analysis revealed that the extraction efficiency of steroids (recoveries ranged from $82 \%$ to $112 \%$ ) was higher with $\beta$-CD than with the Oasis HLB cartridge. ${ }^{52}$ Inclusion complex is a chemical species comprising guest molecules in which one of the host molecules can admit a guest component into its cavity, resulting in a stable encapsulation with chemical and physical factors determining complex formation. Among the steroids bound by entrapped $\beta-C D$ polymers, hydroxylated estrogens exhibited better inclusion complex formation, which may indicate the importance of hydrogen bonding between the phenolic hydroxyl group and the exterior hydroxyl group of $\beta$-CD (Figure 1). In the retention of polar compounds in SPE with polymeric sorbents, the specific surface area and

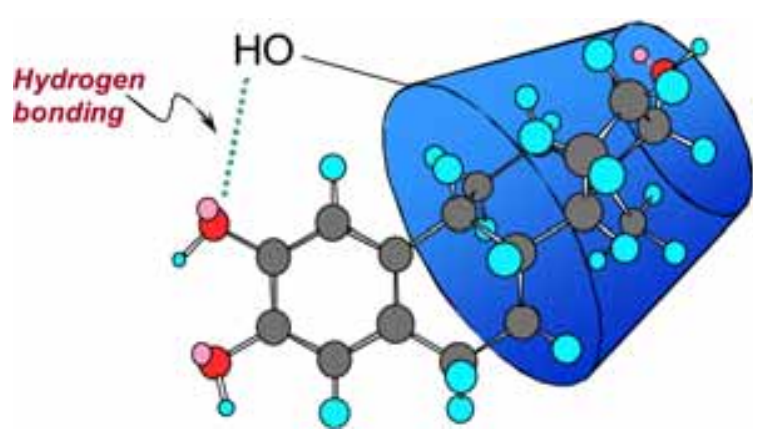

Figure 1. A proposed inclusion complex of $\beta$-cyclodextrin ( $\beta$ CD) with hydroxylated estrogens. Some parts of the molecule are incorporated into the $\beta-C D$ cavity, and others remain on the outside. The A-ring of the steroid molecule predominantly forms complexes with the $\beta-\mathrm{CD}$, and its structural feature greatly influences the stability of the inclusion complexes via hydrogen bonding between phenolic hydroxyl and the exterior hydroxyl groups of estrogen and $\beta-\mathrm{CD}$, respectively. Data are taken from reference \#52. 
the hydrophilicity of the resin play important roles, and a suitable sorbent should exhibit a proper balance of these 2 characteristics. ${ }^{53}$

\section{Hydrolysis of steroid conjugates}

To promote urinary excretion, steroids undergo phase II metabolism, which increases the hydrophilic nature of the steroid. This is achieved by conjugation with either glucuronic acid or sulfuric acid. For GC separation, the analyte of interest must be volatile and thermally stable. Given that steroid conjugates fulfill neither criterion, ${ }^{54}$ preparative steps are required to render the conjugated steroid amenable to GC-MS analysis. This involves hydrolysis of the conjugated steroid to produce the free steroid, followed by chemical derivatization to avoid poor chromatographic behavior.

Hydrolysis can be carried out by biological (enzymatic) or chemical (non-enzymatic) methods. Hydrolysis of conjugated steroids is most commonly performed using enzymatic preparations and, to a lesser extent, chemical approaches. Bacterial (E. coli) and mollusc (Helix pomatia) enzymatic sources are most commonly used. The bacterial sources only contain $\beta$-glucuronidase activity to cleave the glucuronide conjugates, whereas the mollusc sources contain both $\beta$-glucuronidase and sulfatase activities; the latter is responsible for sulfate hydrolysis. Although all enzyme sources possess $\beta$-glucuronidase activity, the activity levels and specificities vary. Once a hydrolysis approach is selected, the conditions for hydrolysis, including the amount of enzyme, $\mathrm{pH}$, temperature, and duration of hydrolysis, can vary dramatically. ${ }^{55}$ The $H$. pomatia enzyme with sulfatase activity is the most widely used enzyme preparation, and has the broadest specificity. Hydrolysis at $55^{\circ} \mathrm{C}$ for $3 \mathrm{~h}$ with $\beta$-glucuronidase/arylsulfatase $(50 \mu \mathrm{L})$ from H. Pomatia and $1 \mathrm{~mL}$ of $0.2 \mathrm{M}$ acetate buffer (pH 5.2) is the most widely used hydrolysis condition for a variety of sample types. The incubation time is typically short for hydrolysis with the $E$. coli enzyme as cleavage of glucuronides can occur within minutes. ${ }^{56}$

Ambiguity also exists over whether to hydrolyze pre- or post-extraction. The SPE with entrapped $\beta$-CD polymer was processed after enzymatic hydrolysis to avoid recovery of unconjugated steroids. ${ }^{52}$ Enzymatic hydrolysis with $\beta$ glucuronidase was carried out first during sample preparation, as the extraction yield of $5 \alpha$-dihydrotestosterone (DHT) was slightly increased with SPE after hydrolysis. ${ }^{39}$

A transformation phenomenon of urinary steroids, including androgens, estrogens, corticoids, progestins, and sterols, in the presence of 2 different enzyme systems was elucidated in a GC-MS-based quantitative steroid profiling study. Two enzymatic hydrolysis procedures were performed: (a) For the hydrolysis of both glucuronide and sulfate conjugates, $1 \mathrm{~mL}$ of $0.2 \mathrm{M}$ acetate buffer (pH 5.2) and $50 \mu \mathrm{L}$ of $\beta$ glucuronidase/arylsulfatase solution was used and the resulting mixture was then incubated at $55^{\circ} \mathrm{C}$ for $3 \mathrm{~h}$; (b) to hydrolyze glucuronide conjugates only, $50 \mu \mathrm{L}$ of $\beta$ glucuronidase was used and then incubated at $55^{\circ} \mathrm{C}$ for $1 \mathrm{~h}$. Hydrolysis with $\beta$-glucuronidase of $E$. coli did not lead to by-products and substrates at concentrations $<1 \mathrm{ng} / \mathrm{mL}$ were also detected. ${ }^{38}$ In addition to incomplete sulfate hydrolysis, incubation with the $H$. pomatia enzyme can also lead to steroid conversion or degradation, and artifact formation. $^{57}$

Chemical hydrolysis can be used individually or in combination with an enzyme preparation (commonly $E$. coli). In general, chemical hydrolysis is achieved using acids, with cleavage of the conjugate being strongly influenced by the choice of acid (hydrochloric or sulfuric), acid molarity, temperature, and duration of the reaction. ${ }^{58}$ In addition, chemical hydrolysis has been carried out by solvolysis, which leads to superior steroid recovery compared to hot acid hydrolysis. ${ }^{59}$ Alternatively, methanolysis, which is a variation of solvolysis using trimethylchlorosilane (TMCS) in methanol, has been employed for a wide range of steroid androgens. Cleavage of both glucuronide and sulfated conjugates occurs due to hydrolysis promoted by the generation of hydrochloric acid from TMCS. ${ }^{60}$ Methonolysis was performed in equine urine post-SPE using methanolic $\mathrm{HCl}^{61}$ The methanolysis procedure generates free steroids at least as large as those obtained by solvolysis. ${ }^{62}$ Extraction of steroid molecules from non-invasive human tissue samples, such as hair and nails, requires other techniques. Ultrasonication and alkaline hydrolysis are the most commonly used methods for extracting unconjugated steroids. ${ }^{63,64}$

\section{Chemical derivatization}

Following hydrolysis and extraction, steroid molecules require derivatization prior to GC-MS analysis to improve their volatility, thermal stability, and chromatographic properties. Derivatization of steroids is generally achieved by silylation or acylation, depending on the individual properties of the steroid and the detection system. The choice of derivatization mixture is dependent on the steroids of interest, as co-elution of derivatized steroids may occur. ${ }^{65}$

\section{Oximation}

Carboxylic acids were commonly esterified by reacting with diazomethane; however, silylation is now more common. ${ }^{66-68}$ Diazomethane in diethyl ether $(0.5 \mathrm{~mL})$ was used to esterify steroid glucuronides from biological extracts followed by silylation with N-methyl-N-trifluoroacetamide (MSTFA)/TMCS (100:1 v/v). ${ }^{33}$

\section{Silylation}

A number of silylating agents are used for the protection of active hydrogens in steroid molecules. In general, the sterically least hindered functional groups containing active hydrogen are the most readily derivatized; however, these are also the most sensitive to hydrolysis with acid or base. Trimethylsilyl (TMS) derivatization is extensively used to derivatize most steroid functional groups, including aliphatic 
and phenolic alcohols and carbonyl and amine groups, to increase volatility for GC-MS. The most common reagents are N,O-bis (trimethylsilyl)-trifluoroacetamide (BSTFA) and the more volatile MSTFA. In an equine urinary profiling of testosterone and ethisterone, oximation was performed using hydroxyamine $\mathrm{HCl}$ in dry pyridine $(10 \% \mathrm{w} / \mathrm{v} ; 50 \mu \mathrm{L})$. Subsequent tert-butyldimethylsilylation (TBDMS) was performed with $5 \%$ diethylamine $\mathrm{HCl}$ in DMF and $\mathrm{N}$-methyl, N-tert.-butyldimethylsilyltri-fluoroacetamide (MTBSTFA). The oxime-TBDMS derivatization showed good specificity and sensitivity for the quantification of ethisterone and testosterone. $^{62}$

The silylation potential of a ketone moiety under steric hindrance can be increased with catalysts. TMS imidazole and trimethyliodosilane (TMIS) are recommended as catalysts. As an alternative reagent, a mixture of MSTFA, ethanethiol, and ammonium iodide $\left(\mathrm{NH}_{4} \mathrm{I}\right)$ has been used in steroid profiling. ${ }^{69}$ Replacement of ethanethiol with dithioerythreitol (DTE) and preparation of the resultant MSTFA: $\mathrm{NH}_{4} \mathrm{I}$ :DTE mixture in desired ratios is in wide practice in various GC-MS-based steroid analysis studies.

For chemical transformation of multi-functional steroids, mixed derivatization is performed to improve physical-

\section{2-OH-E1}

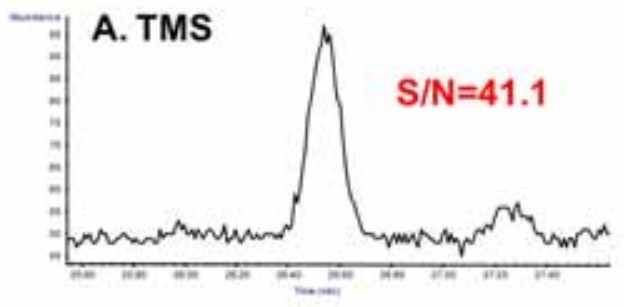

B. EOC-TMS

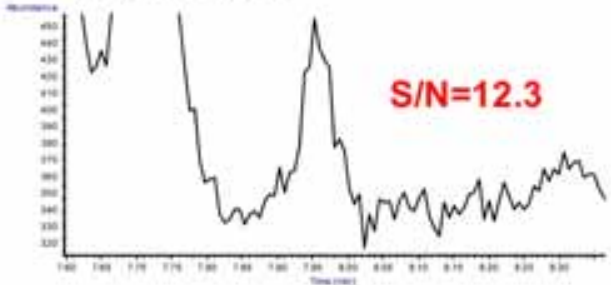

C. EOC-PFPA

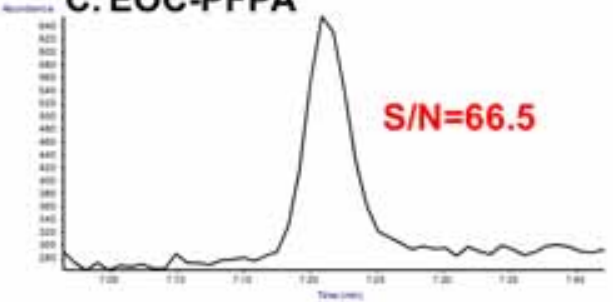

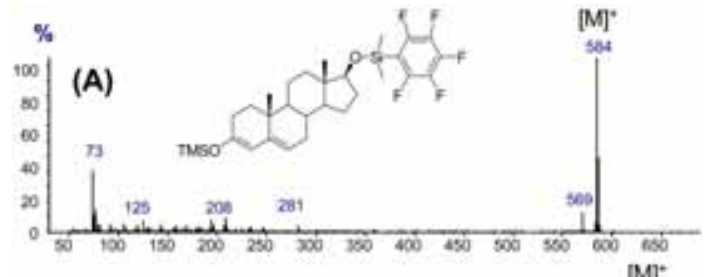
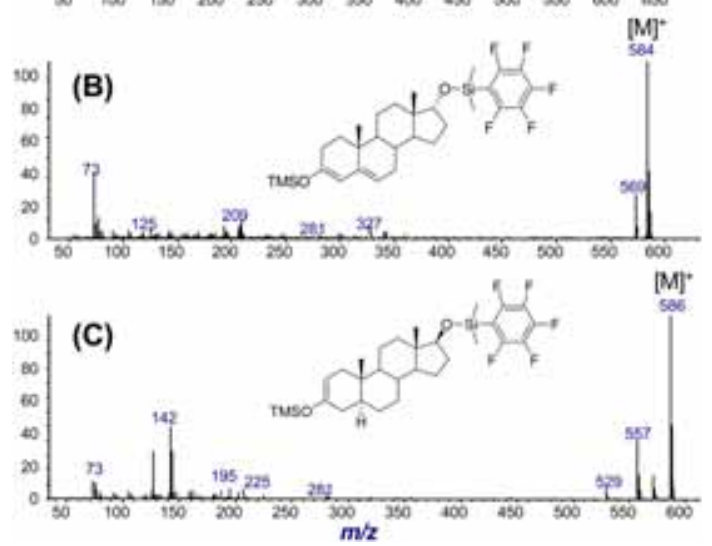

Figure 2. Mass spectra of 3-trimethylsilyl-17-flophemesyl derivatives of (A) testosterone, (B) epitestosterone, and (C) 5adihydrotestosterone. Data are taken from reference $\# 47$.

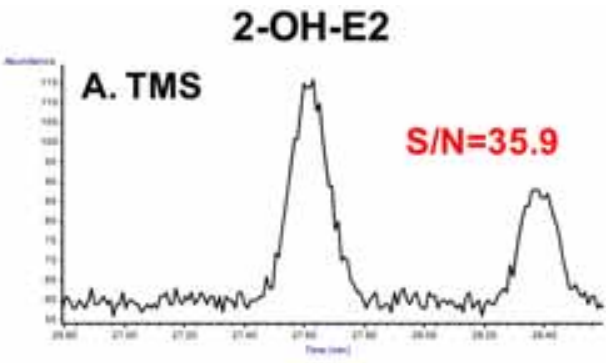

\section{B. EOC-TMS}

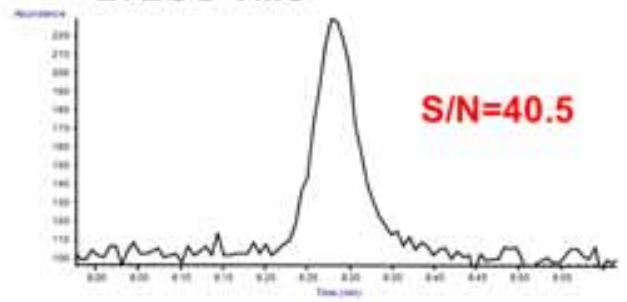

C. EOC-PFPA

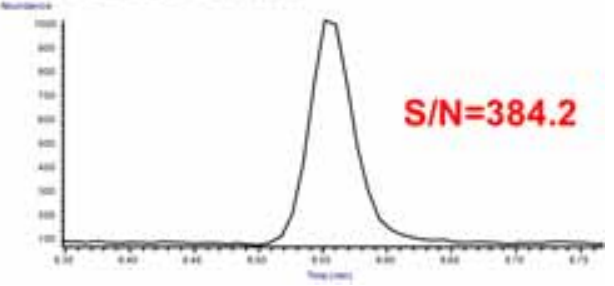

Figure 3. Detectabilities of 3 different derivatization techniques. Nineteen estrogen metabolites were spiked at a urinary concentration of $1 \mathrm{ng} / \mathrm{mL}$ and analyzed. Representative extracted-ion chromatograms based on the quantitative ions of the individual compounds catechol estrone (2-hydroxy-estrone: 2-OH-E1) and estradiol (2-hydroxy-17 $\beta$-estradiol: 2-OH-E2) are presented without smoothing. Data are taken from reference \#73. 
chemical properties and mass spectral characteristics. Sensitive and selective quantification of testosterone and epitestosterone in equine urine was achieved by a combination of TMS and pentafluorophenyldimethylsilylation. ${ }^{63}$ The pentafluorophenyl ring is a strong electronattracting group, which is able to influence the mode of fragmentation of steroid derivatives under electron impact in a way that leads to diagnostic mass spectra. The spectra of pentafluorophenyldimethylsilyl (flophemesyl) derivatives generally display a strong molecular ion and provide more detailed diagnostic information (Figure 2). ${ }^{70-72}$

\section{Acylation and ethoxycarbonylation}

Acylation is another commonly used chemical derivatization method for polar hydroxyl, thiol, and amine groups in GCMS-based steroid analysis. It can be formed with acyl anhydride, acyl halide, and acyl amide, but N-methyl-bis (trifluoroacetamide) has been more commonly used in recent. Estrogens have a phenolic hydroxyl and one or more polar groups, such as alcoholic hydroxyl and/or ketone. An optimized two-phase extractive ethoxycarbonylation (EOC) with ethylchloroformate (ECF) was combined with subsequent pentafluoropropionyl (PFP) derivatization to overcome the high polarity and instability of catechol estrogens in a GC-MS-based analysis. The chemical structures of the derivatives of urinary estrogen metabolites were identified and confirmed using mass spectral patterns. ${ }^{48}$ To improve the detectability with good GC-MS properties, perfluoroacylation with PFPA was performed as subsequent derivatization to protect the remaining aliphatic hydroxyl groups. The chromatographic separation of the 19 estrogens as their EOC-PFP derivatives was achieved with excellent peak shapes and higher responses in serum samples (Figure 3). ${ }^{73}$

\section{Conclusion}

Sample preparation plays a very important role in GCMS-based steroid analysis. Here, we discussed major sample preparation techniques and their significance, particularly with respect to 3 aspects: SPE, hydrolysis, and derivatization. We also compared different SPE techniques and hydrolytic enzymes and discussed the various derivatization methods currently in use. A variety of biological samples have been analyzed for identification and quantification of endogenous steroids. The results obtained were based on the type of sample material used, the target metabolites, and the combination of sample preparation steps.

\section{Acknowledgements}

The sections of this review that refer to our own research have been supported by an intramural grant from the Korea Institute of Science and Technology (KIST).

\section{References}

1. Akyuz, S.; Pince, S.; Hekin, N. J. Clin. Pediatr. Dent. 1996, 20, 219.

2. Yagnucci, A. H.; Evans, E. Am. J. Med. 1986, 80, 83.

3. Farta, L. I.; Kushlinskii, N. E. Probl. Endokrinol. Mosk. 1986, 32, 25.

4. Carpene, G.; Vettoretti, A.; Pedini, F.; Rocco, S.; Mantero, F.; Opocher, G. J. Chromatogr. 1991, 553, 201.

5. Shackleton, C. H. J. Steroid Biochem. Mol. Biol. 1993 , 45,127 .

6. Shackleton, C. H.; Chuang, H.; Kim, J.; de la Torre, X.; Segura J. Steroids 1997, 62, 523.

7. Osamu, N. J. Chromatogr. A 2001, 935, 267.

8. Krone, N.; Hughes, B. A.; Lavery, G. G.; Stewart, P. M.; Arlt, W.; Shackleton, C. H. L. J. Steroid Biochem. Mol. Biol. 2010, 121, 496.

9. Lee, S. H.; Woo, H. M.; Jung, B. H.; Lee, J.; Kwon, O. S.; Pyo, H. S.; Choi, M. H.; Chung, B. C. Anal. Chem. 2007, 79,610 .

10. Weckwerth, W. Annu. Rev. Plant. Biol. 2003, 54, 669.

11. Kaddurah-Daouk, R.; Kristal, B. S.; Weinshilboum, R. M. Annu. Rev. Pharmacol. Toxicol. 2008, 653, 48.

12. Falkenstein, E.; Tillmann, H. C.; Christ, M.; Feuring, M.; Wehling, M. Pharmacol. Rev. 2000, 52, 513.

13. Bulun, S. E.; Yang, S.; Fang, Z.; Gurates, B.; Tamura, M.; Zhou, J.; Sebastian, S. J. Steroid Biochem. Mol. Biol. 2001, 79, 19.

14. Caulfield, M. P.; Lynn, T.; Gottschalk, M. E.; Jones, K. L.; Taylor, N. F.; Malunowicz, E. M.; Shackleton, C. H.; Reitz, R. E.; Fisher, D. A. J. Clin. Endocrinol. Metab. 2002, 87, 3682.

15. Choi, M. H.; Hahm, J. R.; Jung, B. H.; Chung, B. C. Clin. Chim. Acta 2002, 320, 95.

16. Scott, R. R.; Miller, W. L. Horm. Res. 2008, 69, 266.

17. Simard, J.; Ricketts, M. L.; Gingras, S.; Soucy, P.; Feltus, F. A.; Melner, M. H. Endocr. Rev. 2005, 26, 525.

18. White, P. C.; Speiser, P. W. Endocr. Rev. 2000, 21, 245.

19. Wong, S. L.; Shu, S. G.; Tsai, C. R. J. Formos. Med. Assoc. 2006, 105, 177.

20. Nicholson, J. K.; Lindon, J. C.; Holmes, E. Xenobiotica 1999, 29, 1181.

21. Lindon, J. C.; Holmes, E.; Bollard, M. E.; Stanley, E. G.; Nicholson, J. K. Biomarkers 2004, 9, 1.

22. Griffin, J. L.; Bollard, M. E. Curr. Drug Metabol. 2004, $5,389$.

23. Denkert, C.; Budczies, J.; Kind, T.; Weichert, W.; Tablack, P.; Sehouli, J. Cancer Res. 2006, 66, 10795.

24. Palermo, M.; Gomez-Sanchez, C.; Roitman, E.; Shackleton, C. H. L. Steroids 1996, 6, 583.

25. Homma, K.; Hasegawa, T.; Masumoto, M.; Takeshita, E.; Watanabe, K.; Chiba, H.; Kurosawa, T.; Takahashi, T.; Matsuo, N. Endocrinol. J. 2003, 50, 783.

26. Meffre, D.; Pianos, A.; Liere, P.; Eychenne, B.; Cambourg, A.; Schumacher, M.; Stein, D. G; Guennoun, R. Endocrinology 2007, 148, 2505. 
27. Moon, J. Y.; Jung, H. J.; Moon, M. H.; Chung, B. C.; Choi, M. H. Steroids 2008, 73, 1090.

28. Semmes, O. J. Cancer Epidemiol. Biomarkers Prev. 2004, 13, 1555.

29. Cho, Y. D.; Choi, M. H. Bull. Kor. Chem. Soc. 2006, 27, 1315.

30. Choi, M. H.; Kim, J. Y.; Chung, B. C. Analyst 1999, 124, 675.

31. Pichon, V. J. Chromatogr. A 2000, 885, 195.

32. Thurman, E. M.; Mills, M. S. In: Solid-Phase Extraction. Principles and Practice, Winefordner JD (ed) Wiley, New York, 1998.

33. Choi, M. H.; Chung, B. C. Analyst 2002, 126, 306.

34. Choi, M. H.; Kim, K. R.; Hong, J. K.; Park, S. J.; Chung, B. C. Rapid Commun. Mass Spectrom. 2002, 16, 2221.

35. Labadie, P.; Budzinski, H. Anal. Bioanal. Chem. 2005, 381, 1199.

36. Reddy, S.; Iden, C. R.; Brownawell, B. J. Anal. Chem. 2005, 77, 7032 .

37. Li, A. C.; Junga, H.; Shou, W. Z.; Bryant, M. S.; Jiang, X. Y.; Naidong, W. Rapid Commun. Mass Spectrom. 2004, 18, 2343.

38. Lin, W. C.; Chen, H. C.; Ding, W. H. J. Chromatogr. A 2005, 1065, 279.

39. Higashi, T.; Yamaguchi, A.; Shimada, K.; Koh, E.; Mizokami, A.; Namiki, M. Anal. Bioanal. Chem. 2005, 382, 1035.

40. Choi, M. H.; Kim, J. Y.; Chung, B. C. Analyst 1999, $124,675$.

41. Choi, M. H.; Hahm, J. R.; Jung, B. H.; Chung, B. C. Clin. Chim. Acta 2002, 320, 95.

42. Woo, H. M.; Kim, K. M.; Choi, M. H.; Jung, B. H.; Lee, J.; Kong, G.; Nam, S. J, Kim, S.; Bai, S. W.; Chung, B. C. Clin. Chim. Acta 2009, 400. 63.

43. Moon, J. Y.; Jung, H. J.; Moon, M. H.; Chung, B. C.; Choi, M. H. J. Am. Soc. Mass Spectrom. 2009, 20, 1626.

44. Choi, M. H.; Moon, J. Y.; Cho, S. H.; Chung, B. C.; Lee, E. J. BMC Cancer 2011, 11, 342.

45. Moon, J. Y.; Ha, Y. W.; Moon, M. H.; Chung, B. C.; Choi, M. H. Cancer Epidemiol. Biomarkers Prev. 2010, 19, 388.

46. Lee, S. H.; Choi, M. H.; Lee, Y. W.; Chung, B. C. Mass Spectrom. Lett. 2010, 1, 29.

47. Moon, J. Y.; Kang, S. M.; Moon, M. H.; Hong, J.; Kim, K. T.; Jeong, D. H; Kim, Y. N.; Chung, B. C.; Choi, M. H. J. Chromatogr. B 2011, 879, 3742.

48. Ha, Y. W.; Moon, J. Y.; Jung, H. J.; Chung, B. C.; Choi, M. H. J. Chromatogr. B 2009, 877, 4125.
49. Jung, H. J.; Lee, W. Y.; Yoo, Y. S.; Chung, B. C.; Choi, M. H. Clin. Chim. Acta 2010, 411, 818.

50. Jung, H. J.; Kim, S. J.; Lee, W. Y.; Chung, B. C.; Choi, M. H. Rapid Commun. Mass Spectrom. 2011, 25, 1184.

51. Choi, M. H.; Kim, K. R.; Chung, B. C. Steroids 2000, 65, 54.

52. Moon, J. Y.; Jung, H. J.; Moon, M. H.; Chung, B. C.; Choi, M. H. Steroids 2008, 7 3, 1090.

53. Fontanals, N.; Galia, M.; Marce, R. M.; Borrull, F. Chromatographia 2004, 60, 511.

54. Bean, K. A.; Henion, J. D. J. Chromatogr. B 1997, 690, 65.

55. Gomes, R. L.; Meredith, W.; Snape, C. E.; Sephton, M. A. Anal. Bioanal. Chem. 2009, 393, 453.

56. Ferchaud, V.; Le Bizec, B.; Monteau, F.; André, F.; Courcoux, P. Analyst 2000, 125, 2255.

57. Gomes, R. L.; Meredith, W.; Snape, C. E.; Sephton, M. A. J. Pharm. Biomed. Anal. 2009, 49, 1133.

58. Venturelli, E.; Cavalleri, A.; Secreto, G. J. Chromatogr. B 1995, 671, 363.

59. Muehlbaecher, C. A.; Smith, E. K. Clin. Chem. 1970, 16, 158.

60. Dehennin, L.; Lafarge, P.; Dailly, P.; Bailloux, D.; Lafarge, J. P. J. Chromatogr. B 1996, 687, 85.

61. Tang, P. W.; Crone, D. L. Anal. Biochem. 1989, 182, 289.

62. Kim, J. Y.; Choi, M. H.; Kim, S. J.; Kyong, J. B.; Chung, B. C. J. Vet. Pharmacol. Therap. 2001, 24, 147.

63. Choi, M. H.; Yoo, Y. S.; Chung, B. C. J. Invest. Dermatol. 2001, 116, 57.

64. Choi, M. H.; Yoo, Y. S.; Chung, B. C. J. Chromatogr. B 2001, 754, 495.

65. Van de Kerkhof, D. H. Steroid Profiling in Doping Analysis Universiteit Utrecht, Utrecht, Netherlands, 2001.

66. Poole, C. F. Handbook of Derivatives for Chromatography. Heyden 1977, 152.

67. Evershed, R. P. J. M, eds. Handbook of derivatives for chromatography. 1993, 2, 51.

68. Halket, J. M.; Zaikin, V. G. Eur. Mass Spectrom. 2003, 9, 1.

69. Mussell, C.; Wolff Briche, C. S. J.; Hopley, C.; O Connor, G. Accred Qual Assur. 2007, 12, 469.

70. Poole, C. F.; Zlatkis, A. W.; Sye, F.; Singhawangcha, S. Lipids 1980, 15, 734.

71. Jayasinghe, L. Y.; Marriott, P. J.; Carpenter, P. D.; Nichols, P. D. J. Chromatogr. 1998, 809, 109.

72. Choi, M. H.; Yoo, Y. S.; Chung, B. C. J. Chromatogr. B 2001, 754, 495.

73. Moon, J. Y.; Kim, K. J.; Moon, M. H.; Chung, B. C.; Choi, M. H. J. Lipid Res. 2011, 52, 1595. 